\title{
Pourquoi l'éclosion de la maladie à virus Ebola est-elle si difficile à endiguer?
}

\author{
Semalulu $\mathrm{T}^{1}$, Wong $\mathrm{G}^{2^{*}}$, Kobinger $\mathrm{G}^{2}$, Huston $\mathrm{P}^{3}$ \\ ${ }^{1}$ École de médecine du Nord de I'Ontario, Thunder Bay, Ont. \\ 2Laboratoire national de microbiologie, Agence de la santé publique du Canada, Winnipeg, Man. \\ ${ }^{3}$ Cabinet du sous-ministre adjoint, Agence de la santé publique du Canada, Ottawa, Ont. \\ *Auteur-ressource : gary.wong@phac-aspc.gc.ca
}

\section{Résumé}

L'Afrique de l'Ouest est au cœur d'une éclosion sans précédent de la maladie à virus Ebola; plus de 1000 décès ont été observés jusqu'à maintenant et de nombreux nouveaux cas sont signalés chaque jour. L'Organisation mondiale de la Santé (OMS) a déclaré qu'il s'agissait d'une éclosion en mars 2014; et le 6 août 2014, elle en a fait une urgence de santé publique de portée internationale. D'après les décès signalés et le nombre total de cas signalés à l'OMS en date du 11 août 2014, l'éclosion actuelle a un taux de mortalité global de $55 \%$. Les mesures de lutte contre l'éclosion de la maladie à virus Ebola sont efficaces. Alors pourquoi l'éclosion de la maladie à virus Ebola en Afrique de l'Ouest est-elle si difficile à endiguer? Cette maladie se transmet par les liquides organiques, et s'attaque immédiatement au système immunitaire, puis progressivement aux principaux organes et à la paroi des vaisseaux sanguins. Les principaux pays touchés (la Sierra Leone, la Guinée et le Libéria) sont de petits pays qui ne disposent que de ressources limitées face à des éclosions prolongées, notamment dans les régions rurales. II s'agit là d'un défi d'autant plus important que les travailleurs de la santé eux-mêmes sont à risque de contracter la maladie. À ce jour, le traitement n'est que palliatif (pas curatif), et les stratégies de lutte ont dû faire face à la méfiance, en raison de la crainte et de renseignements erronés. Cependant, des progrès importants sont réalisés - l'intervention internationale relative à la maladie à virus Ebola a pris de l'ampleur, des stratégies de communication ont été mises au point pour lutter contre la peur et la méfiance, et des traitements prometteurs sont en cours d'élaboration, notamment une combinaison de trois anticorps monoclonaux qui a été administrée à deux travailleurs de la santé américains infectés par la maladie à virus Ebola. Le Laboratoire national de microbiologie de l'Agence de la santé publique du Canada appuie le travail de diagnostic des laboratoires de l'Afrique de l'Ouest. De plus, l'Agence travaille avec les provinces et les territoires ainsi qu'avec les principaux intervenants, de manière à s'assurer que le Canada est préparé advenant une importation de la maladie à virus Ebola.

\section{Introduction}

Quatre pays de l'Afrique de l'Ouest sont au cœur d'une éclosion sans précédent de la maladie à virus Ebola. L'Organisation mondiale de la Santé (OMS) a signalé cette éclosion en mars 2014. Le 6 août, se fondant sur les recommandations d'un comité d'urgence, elle a déclaré que l'éclosion actuelle est une urgence de santé publique de portée internationale (USPPI) (1). En date du 11 août 2014, l'OMS a signalé 1848 cas et 1013 décès en Guinée, en Sierra Leone, au Libéria et au Nigeria (1). D'après ces décès signalés et le nombre total de cas signalés, l'éclosion a actuellement un taux de mortalité global de $55 \%$.

Le présent article a pour objectif de résumer les connaissances sur la maladie à virus Ebola, les obstacles pour ce qui est d'endiguer l'éclosion et les progrès réalisés à ce jour, notamment la contribution du Canada dans l'intervention contre l'éclosion. 


\section{Contexte}

La maladie à virus Ebola fait partie de la famille des Filoviridæ, dont la plupart des membres causent une fièvre hémorragique grave chez les humains. II existe cinq espèces : Ebola-Zaïre, Ebola-Soudan, Ebola-Budinbugyo, Ebola-Côte d'Ivoire et Ebola-Reston. Chaque espèce renferme un virus. [Tableau 1] $(2,3)$. 
Tableau 1. Espèces du genre Ebolavirus $<3,4>$

\begin{tabular}{l|l|l|l}
\hline Espèce & Virus & Région & Taux de mortalité \\
\hline Ebola-Zaïre & EBOZ & Afrique & De 60 à $90 \%$ \\
\hline Ebola-Soudan & EBOS & Afrique & De 40 à $60 \%$ \\
\hline Ebola-Budinbugyo & EBOB & Afrique & $25 \%$, selon une éclosion \\
\hline Ebola-Côte d'lvoire & EBOCI & Afrique & $\begin{array}{l}\text { Inconnu; une seule infection connue } \\
\text { en Côte d'Ivoire. }\end{array}$ \\
\hline Ebola-Reston & EBOR & Asie & $\begin{array}{l}\text { N'est pas une cause connue } \\
\text { d'infections mortelles chez l'humain. } \\
\text { Mortel chez les primates non } \\
\text { humains. }\end{array}$ \\
\hline
\end{tabular}

L'éclosion actuelle est causée par une nouvelle variante de la maladie à virus Ebola de souche EBOZ, qui est la plus virulente chez les humains (5).

Le réservoir naturel du virus Ebola est inconnu, mais on croit qu'il s'agit des roussettes (chauve-souris) (6). On sait que le virus Ebola provoque la maladie chez les humains, les primates non humains et d'autres mammifères $(4,7)$. On croit que le virus Ebola s'introduit dans la population humaine par l'exposition aux liquides organiques d'une roussette ou d'un mammifère infecté, en particulier de primates non humains. L'infection humaine par la souche EBOZ du virus a été associée à la chasse et à la transformation de la viande de gibier (8 à 10).

Après une période d'incubation de 2 à 21 jours, une personne infectée par le virus Ebola présente d'abord des symptômes non spécifiques (p. ex. maux de tête, fièvre et douleurs musculaires). Cet état évolue jusqu'à une éruption cutanée, à la diarrhée et aux vomissements, habituellement suivis d'une défaillance de plusieurs organes, d'hémorragies et du décès de la victime. La transmission interhumaine se produit par contact direct avec les liquides organiques et les tissus d'une personne infectée (2). Les personnes les plus à risque d'une infection durant les éclosions sont les membres de la famille et les soignants des personnes infectées, les personnes en contact avec des cadavres au cours des préparations et des rituels entourant les funérailles, ainsi que le personnel de soins de santé, en raison de manquements au protocole de sécurité (p. ex. blessures causées par des piqûres d'aiguilles) (11).

Bien que les options thérapeutiques à ce jour soient limitées, les mesures de lutte contre les éclosions de la maladie sont efficaces en vue de mettre un frein à la transmission si elles sont bien exécutées. Ces mesures comprennent les méthodes barrières et de mise en quarantaine pour la restriction de l'exposition, le dépistage précoce, l'isolement des cas, la recherche de contacts, les stratégies de communication visant à réduire les comportements à risque ainsi que la surveillance épidémiologique $(11,12,13)$.

Les mesures de lutte contre l'éclosion de la maladie à virus Ebola sont efficaces. Pourquoi alors l'éclosion en Afrique de l'Ouest est-elle devenue une éclosion si difficile à endiguer?

\section{Les défis}

\section{Le virus Ebola est muni de mécanismes d'attaque mortels}

Le virus Ebola pénètre dans l'hôte par de petites lésions cutanées et la surface des muqueuses, avec l'aide de sa glycoprotéine (GP) de surface. Lors de son entrée dans les cellules, le virus se réplique, comme le virus de descendance se forme dans la membrane de la cellule hôte, et la cellule infectée est détruite $(14,15,16)$. L'analyse de tissus d'humains et de primates non humains infectés a révélé que la réplication virale se produit 
initialement dans les leucocytes, les cellules épithéliales, les hépatocytes ainsi que dans les cellules spléniques, corticosurrénaliennes et endothéliales (4).

\section{Leucocytes}

Les leucocytes - macrophages, monocytes et cellules dendritiques - sont les principales cellules cibles de l'infection (17), ce qui perturbe gravement la réponse immunitaire. Les macrophages et les monocytes participent à la réponse immunitaire innée et sont la première ligne de défense de l'organisme contre les infections. La mort de monocytes et de macrophages entraîne une libération massive de cytokines, ce qui attire encore plus de macrophages, lesquels seront infectés $(18,19,20)$. S'ensuit une chaîne de réaction positive entre les macrophages et les cytokines qui peut conduire à une réaction inflammatoire dérégulée ou un choc cytokinique $(3,19,20,23)$.

Après leur mort, les cellules dendritiques infectées ne peuvent plus activer la réaction immunitaire adaptative. Les patients atteints de la maladie à virus Ebola de souche EBOZ mortelle ne présentent presque aucun anticorps spécifique de l'antigène viral, en raison de l'inhibition de l'immunité lymphocytaire $B$ et $T$ (21). Cela est causé par les cytokines inflammatoires libérées par les macrophages, notamment l'interleukine-10 (IL-10) (22). Ainsi, la souche $E B O Z$ hyperstimule la réponse immunitaire innée et supprime la réponse immunitaire adaptative.

\section{Cellules épithéliales, hépatiques, spléniques et surrénaliennes}

On croit que les leucocytes infectés propagent le virus dans l'organisme par l'intermédiaire du système lymphatique et du sang. Le virus s'attaque alors de préférence aux cellules épithéliales, hépatiques, spléniques et surrénaliennes (4). Les cellules épithéliales infectées qui tapissent la muqueuse intestinale causent les symptômes gastro-intestinaux qui se manifestent aux stades précoces de l'infection (c.-à-d. les vomissements et la diarrhée) (4). Les hépatocytes infectés font augmenter les taux d'enzymes hépatiques et nuisent au fonctionnement du foie. Cela peut diminuer la synthèse des facteurs de la coagulation et contribuer à l'apparition d'anomalies de la coagulation (24). Les cellules spléniques infectées peuvent causer une nécrose et une hémorragie dans la cavité abdominale. La nécrose des cellules corticosurrénaliennes altère la régulation de la tension artérielle et semble contribuer au choc septique qui se produit durant les derniers stades de l'infection (25). Le virus finit par atteindre tous les organes vitaux, ce qui mène à une insuffisance polyviscérale progressive et à un état de choc $(4,20)$.

\section{Cellules endothéliales}

Les cellules endothéliales qui tapissent les vaisseaux sanguins sont ciblées au cours des derniers stades de l'infection. On pense que l'atteinte endothéliale accroît la perméabilité vasculaire, pouvant causer des hémorragies qui constituent une caractéristique importante de l'infection chez de 40 à $50 \%$ des patients $(5,14)$.

Les liens entre cette pathologie et ses signes et symptômes cliniques sont mis en évidence dans le Tableau 2.

Tableau 2. Physiopathologie et signes et symptômes cliniques de l'infection par le virus Ebola

\begin{tabular}{|l|l|l|}
\hline & Signes et symptômes cliniques & Physiopathologie \\
\hline $\begin{array}{l}\text { Symptômes } \\
\text { précoces }\end{array}$ & $\begin{array}{l}\text { Apparition soudaine d'une fièvre, de frissons, } \\
\text { d'un malaise, d'une myalgie } \\
\text { Mal de gorge intense }\end{array}$ & $\begin{array}{l}\text { Les macrophages et les monocytes infectés } \\
\text { libèrent des cytokines }\end{array}$ \\
\hline Première semaine & Généraux : Prostration, léthargie & $\begin{array}{l}\text { Les cytokines contribuent aux symptômes } \\
\text { généraux }\end{array}$ \\
\cline { 2 - 3 } & $\begin{array}{l}\text { Gastro-intestinaux : Anorexie, nausées, } \\
\text { vomissements, douleurs abdominales, } \\
\text { diarrhée (ainsi qu'une apparition progressive } \\
\text { d'une diarrhée sanglante et d'une }\end{array}$ & $\begin{array}{l}\text { La réplication virale dans les cellules } \\
\text { symptiales et endothéliales provoque des } \\
\text { hémorragies }\end{array}$ \\
\hline
\end{tabular}




\begin{tabular}{|c|c|c|}
\hline & hématémèse) & \\
\hline & $\begin{array}{l}\text { Cardiaques : Douleur thoracique, dyspnée, } \\
\text { essoufflement, toux, écoulement nasal }\end{array}$ & $\begin{array}{l}\text { Réplication virale et nécrose des tissus } \\
\text { cardiaques }\end{array}$ \\
\hline & $\begin{array}{l}\text { Spléniques : Fièvre, douleurs abdominales, } \\
\text { hémorragie (s'il y a rupture dans la cavité } \\
\text { péritonéale) }\end{array}$ & Réplication virale et nécrose \\
\hline & $\begin{array}{l}\text { Hépatiques : Taux élevé d'enzymes } \\
\text { hépatiques et anomalies de la coagulation }\end{array}$ & $\begin{array}{l}\text { Les cellules hépatiques sont infectées, ce } \\
\text { qui entraîne leur mort; cela influe sur la } \\
\text { production de facteurs de la coagulation }\end{array}$ \\
\hline & $\begin{array}{l}\text { Vasculaires : Hyperhémie conjonctivale, } \\
\text { hypotension orthostatique, œdème }\end{array}$ & $\begin{array}{l}\text { Les cellules endothéliales sont déréglées et } \\
\text { des cytokines sont libérées, ce qui augmente } \\
\text { la perméabilité vasculaire }\end{array}$ \\
\hline & $\begin{array}{l}\text { Neurologiques : Maux de tête, confusion, } \\
\text { encéphalite, convulsions, coma }\end{array}$ & $\begin{array}{l}\text { Réplication virale dans le tissu cérébral et } \\
\text { dysfonctionnement vasculaire }\end{array}$ \\
\hline & $\begin{array}{l}\text { Cutanés : Éruption cutanée maculopapulaire } \\
\text { avec divers degrés d'érythème et de } \\
\text { desquamation }\end{array}$ & Fuite endothéliale \\
\hline \multirow[t]{2}{*}{ Complications } & $\begin{array}{l}\text { Hémorragie } \\
\text { Pétéchies, ecchymoses, hémorragies non } \\
\text { maîtrisées aux points de ponction veineuse, } \\
\text { épistaxis, épanchements hémorragiques } \\
\text { viscéraux et autres hémorragies des } \\
\text { muqueuses }\end{array}$ & $\begin{array}{l}\text { L'infection des cellules hépatiques provoque } \\
\text { une hausse du taux d'enzymes hépatiques } \\
\text { et des anomalies de la coagulation } \\
\text { Les lésions des cellules endothéliales } \\
\text { entraînent une augmentation de la } \\
\text { perméabilité vasculaire }\end{array}$ \\
\hline & $\begin{array}{l}\text { État de choc et hypotension } \\
\text { Troubles métaboliques graves } \\
\text { Coagulation intravasculaire disséminée et } \\
\text { choc hypovolémique }\end{array}$ & $\begin{array}{l}\text { Les dommages viraux directs des tissus et } \\
\text { des organes peuvent provoquer la } \\
\text { défaillance d'un organe et un état de choc } \\
\text { Les cellules surrénaliennes infectées } \\
\text { n'arrivent plus à réguler la tension artérielle, } \\
\text { ce qui cause une hypotension et un choc } \\
\text { septique } \\
\text { Coagulopathie diffuse }\end{array}$ \\
\hline $\begin{array}{l}\text { Résultats de } \\
\text { laboratoire }\end{array}$ & $\begin{array}{l}\text { Leucopénie précoce, lymphopénie et } \\
\text { neutrophilie subséquente, thrombocytopénie, } \\
\text { prolongation du temps de Quick et du temps } \\
\text { de céphaline activé } \\
\text { Taux élevés d'aminotransférase sérique } \\
\text { Hyperprotéinémie et protéinurie }\end{array}$ & $\begin{array}{l}\text { L'infection des cellules dendritiques nuit à la } \\
\text { réponse immunitaire } \\
\text { Régulation positive incontrôlée de la } \\
\text { sécrétion de cytokines et de chimiokines } \\
\text { (choc cytokinique) } \\
\text { Réplication virale étendue et mort des } \\
\text { cellules spléniques, rénales, hépatiques, } \\
\text { gonadiques, etc. }\end{array}$ \\
\hline
\end{tabular}

\section{L'élimination du réservoir n'est pas possible}

On ignore l'ampleur et la prévalence du réservoir EBOZ chez les animaux sauvages, d'où l'impossibilité de prévenir les cas sporadiques de transmission d'animal à humain. 


\section{Le traitement est palliatif, pas curatif}

II n'existe actuellement aucun traitement thérapeutique approuvé pour la maladie à virus Ebola. Jusqu'à tout récemment, le traitement reposait sur la réhydratation, la gestion des électrolytes, la prise d'antibiotiques et d'antiviraux pour traiter des infections secondaires et la prise de médicaments pour soulager la douleur, la fièvre et les troubles gastro-intestinaux (2).

\section{L'éclosion s'est étendue jusqu'aux zones urbaines}

La souche $\mathrm{EBOZ}$ a été responsable d'éclosions sporadiques dans les régions forestières éloignées de l'Afrique subsaharienne. C'est dans le cadre de cette éclosion que l'on a observé pour la toute première fois la transmission de la souche EBOZ du virus dans une grande ville, importée par une personne infectée au Nigeria, pays le plus peuplé de l'Afrique. La taille et l'emplacement sans précédent de l'éclosion, conjugués au fait que le virus circule maintenant dans des centres urbains à forte densité de population, rendent cette éclosion difficile à endiguer (1).

\section{Les pays touchés font face à des défis de taille en ce qui concerne les infrastructures sanitaires}

La Sierra Leone, la Guinée et le Libéria sont de petits pays qui ne disposent que de ressources limitées face à des éclosions prolongées, notamment dans les régions rurales. C'est la première fois que l'Afrique de l'Ouest est appelée à lutter contre une éclosion causée par le virus Ebola de souche EBOZ; la plupart des agents de soins de santé primaires n'ont donc aucune expérience par rapport à ce virus. La surveillance et les systèmes de déclaration limités ont peut-être retardé la détection de l'éclosion et l'intervention mondiale subséquente. L'OMS a cité ces problèmes parmi les principales lacunes de l'intervention lors de l'éclosion $(1,26)$.

\section{Les travailleurs de la santé à risque d'infection}

L'OMS a signalé que la transmission dans les établissements de santé est un problème central de l'éclosion actuelle (1). Les travailleurs de la santé courent un grand risque de contracter le virus Ebola de souche EBOZ en s'exposant de façon accidentelle à des liquides organiques lors de soins aux patients infectés. À ce jour, plus de 170 travailleurs de la santé ont été infectés et au moins 81 en sont décédés (27).

Ces décès ont dissuadé des travailleurs de la santé et des organisations internationales de participer aux efforts de traitement et de contrôle du virus (1).

\section{Les stratégies de maîtrise de l'éclosion ont été accueillies par la méfiance}

On a cité la résistance persistante au sein des collectivités comme étant un défi important au chapitre de l'intervention du secteur de la santé en réponse à l'éclosion (1). Des stratégies efficaces de prévention et de contrôle des infections ont été minées par la peur, la méfiance et la désinformation dans les collectivités touchées, ce qui a mené des personnes à croire que le personnel médical avait introduit le virus aux pays atteints. Cet état de choses a fait en sorte que certaines personnes ont refusé de collaborer avec le personnel médical, ont aidé des patients à s'échapper des salles d'isolement et ont adopté des comportements hostiles $(1,26,28)$. Les pratiques traditionnelles d'inhumation présentent également un risque important pour les proches, comme elles peuvent généralement comporter le lavage et le frottement de cadavres pouvant présenter une charge élevée du virus Ebola. La recommandation préconisant que de telles pratiques soient effectuées par des membres de l'équipe d'intervention a été perçue comme étant en conflit avec les croyances et les pratiques culturelles (26). 


\section{Progrès à ce jour}

\section{L'intervention internationale s'affermit}

La communauté internationale envoie de plus en plus de ressources et d'aide en Afrique de l'Ouest afin d'endiguer l'éclosion de la maladie à virus Ebola de souche EBOZ. L'OMS coordonne les efforts visant à accroître les ressources humaines et financières requises pour mener des activités efficaces de prévention et de contrôle des infections. Cela permettra également de mettre en place l'infrastructure nécessaire pour gérer les éclosions futures, notamment le renforcement des capacités de surveillance et des capacités des services de laboratoire. De nombreuses organisations non gouvernementales, dont Médecins sans frontières, Aide à l'enfance et des organisations religieuses, travaillent sur le terrain pour aider à freiner la progression de cette maladie (1).

À la lumière des délibérations d'un comité d'urgence, la directrice générale de l'OMS a formulé un certain nombre de recommandations, notamment : les États touchés doivent déclarer l'éclosion comme étant une urgence nationale et mettre sur pied un centre d'opérations d'urgence en vue de coordonner les efforts d'intervention et de soutien; un dépistage à la sortie du pays doit être réalisé dans tous les aéroports internationaux, ports maritimes et principaux points de passages entre pays afin de repérer les personnes présentant une maladie fébrile inexpliquée; il faut assurer une prise en charge adéquate des contacts; tous les États doivent renforcer leur capacité à détecter, à examiner et à gérer les cas de maladie à virus Ebola grâce à l'amélioration de la surveillance, au soutien du diagnostic en laboratoire et à une intervention rapide (29).

Le Canada collabore avec d'autres pays et organismes ainsi qu'avec l'OMS en offrant un soutien financier et technique. En date du 8 août 2014, le Canada a contribué pour plus de cinq millions de dollars en vue de soutenir les interventions humanitaires, de contrôle des infections et de sécurité en Afrique de l'Ouest (30). En tant que membre du Réseau mondial d'alerte et d'action en cas d'épidémie de l'OMS, le Canada fournit des ressources techniques et humaines pour la détection et l'intervention relatives aux éclosions importantes à l'échelle internationale (31).

Le Laboratoire national de microbiologie (LNM) de l'Agence de la santé publique du Canada (l'Agence) a travaillé en étroite collaboration avec l'OMS en vue de fournir un soutien rapide en matière de diagnostic dans des laboratoires mobiles en Sierra Leone (32).

Au Canada, l'Agence dirige les efforts de préparation du pays à la gestion des cas d'infection au virus Ebola, travaillant en étroite collaboration avec les provinces, les territoires et tous les intervenants concernés. Sont présentement mis à jour bon nombre de dispositifs : définitions de cas, lignes directrices visant le contrôle de l'infection, directives de prise en charge par la santé publique des cas et des contacts associés à la maladie à virus Ebola, lignes directrices en matière de décontamination de l'environnement et de biosécurité, etc. L'Agence aide également à l'élaboration de lignes directrices relatives aux soins cliniques en collaboration avec l'Association pour la microbiologie médicale et l'infectiologie Canada, la Société canadienne de soins intensifs et l'Association canadienne des médecins d'urgence.

\section{Mise en œuvre de stratégies de communication en vue d'apaiser les craintes et de dissiper les idées fausses}

Une évaluation de l'éclosion actuelle, dirigée par le $D^{r}$ Luis Sambo, directeur régional de l'OMS pour l'Afrique, a recommandé aux gouvernements touchés d'accroître les ressources nationales visant à promouvoir le changement des comportements, tout en respectant les pratiques culturelles (1). Des collaborations locales (p. ex. formation des membres des collectivités pour repérer les contacts et collaboration avec des dirigeants locaux pour diffuser efficacement les bons renseignements sur le virus Ebola de souche EBOZ) servent à dissiper les idées fausses et à renforcer les stratégies de contrôle (26). Des collaborations avec les dirigeants des groupes religieux, des collectivités et des tribus servent à diffuser l'information $(26,28)$. Ces messages sont également télédiffusés et radiodiffusés (33). 


\section{Traitements expérimentaux en cours d'élaboration}

Plusieurs traitements expérimentaux sont en cours d'élaboration $(2,34)$. On tente ainsi de mettre au point un traitement pouvant offrir une protection croisée. Le médicament expérimental a été administré à deux Américains infectés par le virus; le médicament en question comprend trois anticorps monoclonaux chimériques murinshumains fabriqués à partir de la plante Nicotiana benthamiana (35). Ces anticorps ont assuré une protection intégrale contre le virus Ebola de souche EBOZ chez des macaques cynomolgus infectés (36).

Un autre traitement expérimental utilise de petits $A R N$ interférents propres à certains gènes du virus Ebola de souche $\mathrm{EBOZ}$ pour inhiber la réplication du virus. Une étude a révélé une protection à $66 \%$ et à $100 \%$ contre le virus Ebola de souche EBOZ chez des macaques, après quatre et sept traitements post-exposition, respectivement (37).

Plusieurs vaccins expérimentaux semblent prometteurs pour contrer le virus Ebola de souche EBOZ chez les primates non humains, y compris un vaccin à base d'adénovirus et un vaccin à base du virus de la stomatite vésiculaire. Par exemple, le vaccin à base du virus de la stomatite vésiculaire a démontré protection immunitaire élevée contre la maladie à virus Ebola de souche EBOZ, et n'a été associé à aucun effet indésirable perceptible chez les primates non humains (38-40). On accélère actuellement les démarches pour que le vaccin à base d'adénovirus et le vaccin à base du virus de la stomatite vésiculaire contre le virus Ebola fassent tous deux l'objet d'essais cliniques de phase I.

Le recours à des traitements expérimentaux à base d'anticorps chez deux Américains infectés par le virus Ebola de souche EBOZ a incité l'OMS à tenir des discussions en vue d'examiner les considérations éthiques concernant l'inclusion de tels traitements dans les efforts d'intervention $(1,41)$.

\section{Conclusion}

Nombre de facteurs font qu'il est difficile d'endiguer l'éclosion du virus Ebola en Afrique de l'Ouest. Le virus réside dans un réservoir faunique peu connu et a émergé dans des pays qui éprouvent des difficultés tant pour ce qui est des capacités de soins de santé que de la communication des risques. Ces différents facteurs se sont manifestés dans un contexte de déplacements mondiaux croissants.

Le Canada participe à part entière à l'intervention mondiale grâce à un éventail de moyens : contributions financières, soutien de laboratoire, travaux pionniers en matière de développement de vaccins et de traitement post-exposition, ainsi que de collaborations internationales. L'Agence de la santé publique du Canada pour sa part coordonne l'intervention nationale de façon à assurer une efficacité optimale concernant tous cas potentiels d'Ebola à l'intérieur de nos frontières en ce qui concerne la détection, la recherche, la gestion et l'établissement de rapports.

L'OMS a déclaré que l'éclosion d'Ebola était une urgence de santé publique de portée internationale. Cette situation continuera à exiger une collaboration nationale et internationale, ainsi qu'une vigilance constante de la part des professionnels des soins de santé de première ligne et de la santé publique, de façon à mettre un terme à l'éclosion du virus en Afrique de l'Ouest et, ainsi, à prévenir sa propagation au niveau mondial.

\section{Remerciements}

La $D^{\text {re }}$ Huston est la rédactrice scientifique du Relevé des maladies transmissibles au Canada et se récuse des décisions de la rédaction relatives au présent article. Mille mercis au $D^{r}$ Tom Wong d'avoir assumé le rôle de rédacteur en chef de cette communication rapide. Nous tenons également à remercier Mike Drebot et Tim Booth 
d'avoir contribué à la préparation du présent article, ainsi que les évaluateurs qui ont fourni une rétroaction utile et opportune tout en participant aux efforts d'intervention.

\section{Références}

(1) Organisation mondiale de la Santé. Alerte et action au niveau mondial : Maladie à virus Ebola [Internet]. Organisation mondiale de la Santé [consulté en 2014]. Accès :

http://www.who.int/csr/don/archive/disease/ebola/fr/

(2) Organisation mondiale de la Santé. Maladie à virus Ebola : informations générales et résumé de la situation en Afrique de l'Ouest [Internet]. Organisation mondiale de la Santé [consulté en juillet 2014]. Accès : http://www.who.int/csr/don/2014_04_ebola/fr/

(3) Wong G, Kobinger GP et Qiu X. Characterization of host immune responses in Ebola virus infections. Expert Rev Clin Immunol. Juin 2014;10(6):781-90.

(4) Feldmann H et Geisbert TW. Ebola haemorrhagic fever. Lancet [Internet]. 5 mars 2011;377(9768):849-62.

(5) Baize S, Pannetier D, Oestereich L, Rieger T, Koivogui L, Magassouba N, Soropogui B, Sow MS, Keita S, De Clerck H, Tiffany A, Dominguez G, Loua M, Traore A, Kolie M, Malano ER, Heleze E, Bocquin A, Mely S, Raoul H, Caro V, Cadar D, Gabriel M, Pahlmann M, Tappe D, SchmidtChanasit J, Impouma B, Diallo AK, Formenty P, Van Herp M et Gunther S. Emergence of Zaire Ebola Virus Disease in Guinea - Preliminary Report. N Engl J Med. 16 avril 2014.

(6) Leroy EM, Kumulungui B, Pourrut X, Rouquet P, Hassanin A, Yaba P, Delicat A, Paweska JT, Gonzalez JP et Swanepoel R. Fruit bats as reservoirs of Ebola virus. Nature. $1^{\mathrm{er}}$ déc. 2005;438(7068):575-6.

(7) Weingartl HM, Nfon C et Kobinger G. Review of Ebola virus infections in domestic animals. Dev Biol (Basel). 2013;135:211-8.

(8) Leroy EM, Rouquet P, Formenty P, Souquiere S, Kilbourne A, Froment JM, Bermejo M, Smit S, Karesh W, Swanepoel R, Zaki SR et Rollin PE. Multiple Ebola Virus Transmission Events and Rapid Decline of Central African Wildlife. Science. 16 janv. 2004;303(5656):387-90.

(9) Nkoghe D, Kone ML, Yada A et Leroy E. A limited outbreak of Ebola haemorrhagic fever in Etoumbi, Republic of Congo, 2005. Trans R Soc Trop Med Hyg. Août 2011;105(8):466-72.

(10) Georges-Courbot MC, Sanchez A, Lu CY, Baize S, Leroy E, Lansout-Soukate J, Tevi-Benissan C, Georges AJ, Trappier SG, Zaki SR, Swanepoel R, Leman PA, Rollin PE, Peters CJ, Nichol ST et Ksiazek TG. Isolation and phylogenetic characterization of Ebola viruses causing different outbreaks in Gabon. Emerg Infect Dis [Internet]. Janv.-mars 1997;3(1):59-62.

(11) MacNeil A et Rollin PE. Ebola and Marburg Hemorrhagic Fevers: Neglected Tropical Diseases? PLoS Negl Trop Dis. Juin 2012;6(6):e1546.

(12) Raabe VN, Borcherta M. Infection control during filoviral hemorrhagic Fever outbreaks. J Glob Infect Dis. 2012;4(1):69-74.

(13) Organisation mondiale de la Santé. Case definition recommendations for Ebola or Marburg Virus Diseases [Internet]. Genève, Suisse : Organisation mondiale de la Santé, avril 2014 [consulté en août 2014]; [3]. Accès : http://www.who.int/csr/resources/publications/ebola/ebola-casedefinition-contact-en.pdf?ua=1 
(14) Hoenen T, Groseth A, Falzarano D et Feldmann H. Ebola virus: unravelling pathogenesis to combat a deadly disease. Trends Mol Med. Mai 2006;12(5):206-15.

(15) Sullivan N, Yang ZY et Nabel GJ. Ebola Virus Pathogenesis: Implications for Vaccines and Therapies. J Virol. Sept. 2003;77(18):9733-7.

(16) Beniac DR, Melito PL, Devarennes SL, Hiebert SL, Rabb MJ, Lamboo LL, Jones SM et Booth TF. The Organisation of Ebola Virus Reveals a Capacity for Extensive, Modular Polyploidy. PLoS One. 2012;7(1):e29608.

(17) Geisbert TW, Young HA, Jahrling PB, Davis KJ, Larsen T, Kagan E et Hensley LE. Pathogenesis of Ebola hemorrhagic fever in primate models: evidence that hemorrhage is not a direct effect of virus-induced cytolysis of endothelial cells. Am J Pathol. Déc. 2003;163(6):2371-82.

(18) Bray M et Geisbert TW. Ebola virus: the role of macrophages and dendritic cells in the pathogenesis of Ebola hemorrhagic fever. Int J Biochem Cell Biol. Août 2005;37(8):1560-6.

(19) Feldmann H, Bugany H, Mahner F, Klenk HD, Drenckhahn D et Schnittler HJ. Filovirusinduced endothelial leakage triggered by infected monocytes/macrophages. J Virol. Avril 1996;70(4):2208-14.

(20) Zampieri CA, Sullivan NJ et Nabel GJ. Immunopathology of highly virulent pathogens: insights from Ebola virus. Nat Immunol. Nov. 2007;8(11):1159-64.

(21) Wong G, Richardson JS, Pillet S, Patel A, Qiu X, Alimonti J, Hogan J, Zhang Y, Takada A, Feldmann $\mathrm{H}$ et Kobinger GP. Immune Parameters Correlate with Protection Against Ebola Virus Infection in Rodents and Nonhuman Primates. Sci Transl Med. 31 oct. 2012;4(158):158ra146.

(22) Said EA, Dupuy FP, Trautmann L, Zhang Y, Shi Y, El-Far M, Hill BJ, Noto A, Ancuta P, Peretz Y, Fonseca SG, Van Grevenynghe J, Boulassel MR, Bruneau J, Shoukry NH, Routy JP, Douek DC, Haddad EK et Sekaly RP. Programmed death-1-induced interleukin-10 production by monocytes impairs CD4+ T cell activation during HIV infection. Nat Med. Avril 2010;16(4):452-9.

(23) Wauquier N, Becquart P, Padilla C, Baize S et Leroy EM. Human Fatal Zaire Ebola Virus Infection Is Associated with an Aberrant Innate Immunity and with Massive Lymphocyte Apoptosis. PLoS Negl Trop Dis. 5 oct. 2010;4(10):10.1371/journal.pntd.0000837.

(24) Geisbert TW, Jahrling PB, Hanes MA et Zack PM. Association of Ebola-related Reston virus particles and antigen with tissue lesions of monkeys imported to the United States. J Comp Pathol. Févr. 1992;106(2):137-52.

(25) Ryabchikova EI, Kolesnikova LV et Luchko SV. An Analysis of Features of Pathogenesis in Two Animal Models of Ebola Virus Infection. J Infect Dis. Févr. 1999;179 Suppl 1:S199-202.

(26) Andrew Green. Ebola emergency meeting establishes new control centre. Lancet. 2014;384:118.

(27) Thomson Reuters. Ebola outbreak: High death toll among health worker 1st responders [Internet]. CBC News, le 9 août 2014 [consulté en août 2014]. Accès :

http://www.cbc.ca/news/health/ebola-outbreak-high-death-toll-among-health-worker-1stresponders-1.2732076

(28) Organisation mondiale de la Santé. Lutter contre les idées fausses qui entourent la maladie à virus Ebola en Guinée [Internet]. Genève, Suisse : Organisation mondiale de la Santé [consulté en juillet 2014]. Accès : http://www.who.int/features/2014/ebola-myths/fr/

(29) Organisation mondiale de la Santé. Déclaration de l'OMS sur la réunion du Comité d'urgence du Règlement sanitaire international concernant la flambée de maladie à virus Ebola en Afrique de 
l'Ouest en 2014. Genève, Suisse : Organisation mondiale de la Santé, le 8 août 2014 [consulté en août 2014]. Accès : http://who.int/mediacentre/news/statements/2014/ebola-20140808/fr/

(30) Gouvernement du Canada. Document d'information - Réponse du Canada à l'éclosion d'Ebola en Afrique de l'Ouest. Gouvernement du Canada, le 8 août 2014 [consulté en août 2014]. Accès : http://www.international.gc.ca/media/dev/news-communiques/2014/08/08b_bg.aspx?lang=fra

(31) Organisation mondiale de la Santé. Alerte et action au niveau mondial : Réseau mondial d'alerte et d'action en cas d'épidémie. Genève, Suisse : Organisation mondiale de la Santé [consulté en août 2014]. Accès : http://www.who.int/csr/outbreaknetwork/fr/

(32) Agence de la santé publique du Canada. Déclaration du $D^{r}$ Gregory Taylor, administrateur en chef adjoint de la santé publique, sur l'éclosion d'Ebola en Afrique. Agence de la santé publique du Canada, juillet 2014 [consulté en juillet 2014]. Accès : http://www.phac-aspc.gc.ca/cphoacsp/statements/20140730-fra.php

(33) Organisation mondiale de la Santé. Involving everyone: social mobilization is key in an Ebola outbreak response [Internet]. Organisation mondiale de la Santé, mai 2014 [consulté en juillet 2014]. Accès : http://www.who.int/features/2014/social-mobilisation/en/

(34) Leroy EM, Gonzalez JP et Baize S. Ebola and Marburg haemorrhagic fever viruses: major scientific advances, but a relatively minor public health threat for Africa. Clin Microbiol Infect. Juillet 2011;17(7):964-76.

(35) Mapp Biopharmaceutical. ZMapp Information Sheet. San Diego, Californie : Mapp Biopharmaceutical, 2014 [consulté en août 2014]. Accès : http://mappbio.com/zmapinfo.pdf

(36) Qiu X, Audet J, Wong G, Fernando L, Bello A, Pillet S, Alimonti JB et Kobinger GP. Sustained protection against Ebola virus infection following treatment of infected nonhuman primates with ZMAb. Sci Rep. 28 nov. 2013;3:3365.

(37) Geisbert TW, Lee AC, Robbins M, Geisbert JB, Honko AN, Sood V, Johnson JC, de Jong S, Tavakoli I, Judge A, Hensley LE et Maclachlan I. Postexposure protection of non-human primates against a lethal Ebola virus challenge with RNA interference: a proof-of-concept study. Lancet. 29 mai 2010;375(9729):1896-905.

(38) Jones SM, Feldmann H, Ströher U, Geisbert JB, Fernando L, Grolla A, Klenk HD, Sullivan NJ, Volchkov VE, Fritz EA, Daddario KM, Hensley LE, Jahrling PB et Geisbert TW. Live attenuated recombinant vaccine protects nonhuman primates against Ebola and Marburg viruses. Nat Med. Juillet 2005;11(7):786-90. [publié en ligne le 5 juin 2005].

(39) Gunther S, Feldmann H, Geisbert TW, Hensley LE, Rollin PE, Nichol ST, et al. Management of Accidental Exposure to Ebola Virus in the Biosafety Level 4 Laboratory, Hamburg, Germany. $J$ Infect Dis. 2011;204 Suppl 3:S785-90.

(40) Geisbert TW, Daddario-Dicaprio KM, Lewis MG, Geisbert JB, Grolla A, Leung A, Paragas J, Matthias L, Smith MA, Jones SM, Hensley LE, Feldmann H et Jahrling PB. Vesicular Stomatitis Virus-Based Ebola Vaccine Is Well-Tolerated and Protects Immunocompromised Nonhuman Primates. PLoS Pathog. Nov. 2008;4(11). [publié en ligne le 28 novembre 2008].

(41) Organisation mondiale de la Santé. Déclaration : Considérations éthiques liées à l'utilisation d'interventions non homologuées contre la maladie à virus Ebola. 12 août 2014. Accès : http://www.who.int/mediacentre/news/statements/2014/ebola-ethical-review-summary/fr/ 
301| RMTC - Le 14 août 2014 • Volume 40-14 